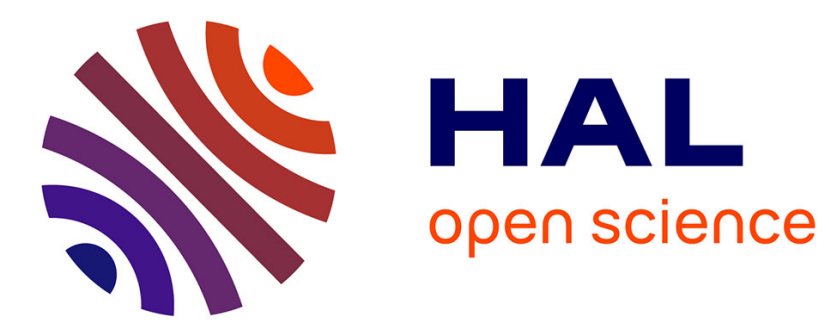

\title{
Short-term wave force prediction for wave energy converter control
}

\author{
Hoaï-Nam Nguyen, Paolino Tona
}

\section{To cite this version:}

Hoaï-Nam Nguyen, Paolino Tona. Short-term wave force prediction for wave energy converter control. Control Engineering Practice, 2018, 75, pp.26-37. hal-01940727

\section{HAL Id: hal-01940727 \\ https://hal-ifp.archives-ouvertes.fr/hal-01940727}

Submitted on 30 Nov 2018

HAL is a multi-disciplinary open access archive for the deposit and dissemination of scientific research documents, whether they are published or not. The documents may come from teaching and research institutions in France or abroad, or from public or private research centers.
L'archive ouverte pluridisciplinaire HAL, est destinée au dépôt et à la diffusion de documents scientifiques de niveau recherche, publiés ou non, émanant des établissements d'enseignement et de recherche français ou étrangers, des laboratoires publics ou privés. 


\title{
Short-term wave force prediction for wave energy converter control
}

\author{
Hoai-Nam Nguyen*†, Paolino Tona ${ }^{\dagger}$ \\ $\dagger$ Control, Signal and System Department - IFP Energies Nouvelles, Rond-point de \\ l'echangeur de Solaize BP3, 69360 Solaize, France \\ hoai-nam.nguyen@ifp.fr,paolino.tona@ifp.fr
}

\begin{abstract}
Given the importance of wave excitation force prediction in most advanced control schemes for wave energy converters, where every new wave force estimation becomes available every fraction of second, the main objective of this paper is to perform a short-term wave prediction that can meet a trade-off between low computational complexity, limited memory usage and accuracy. To this aim, two prediction algorithms are proposed using Kalman filtering theory. The proposed prediction methods are evaluated by using real measurements.

Keywords: Wave Force Prediction, Extended Kalman Filter, Adaptive Kalman Filter, Model Predictive Control, Wave Energy Converter
\end{abstract}

\section{INTRODUCTION}

Wave energy converters (WECs) are devices used to produce electrical energy from wave movements. A schematic example of a WEC is given in Figure 1 an oscillating body (the captor or primary converter) moves under the action 5 of waves and is connected to a Power-Take-Off (PTO) system; the PTO, by exercising an appropriate force on the captor, converts its mechanical energy into electrical energy. The PTO can be a linear electric generator, or a multistage device, such as a hydraulic motor connected to a rotary electric generator.

${ }^{*}$ Corresponding author

Preprint submitted to Control Engineering Practice

July 13, 2018 


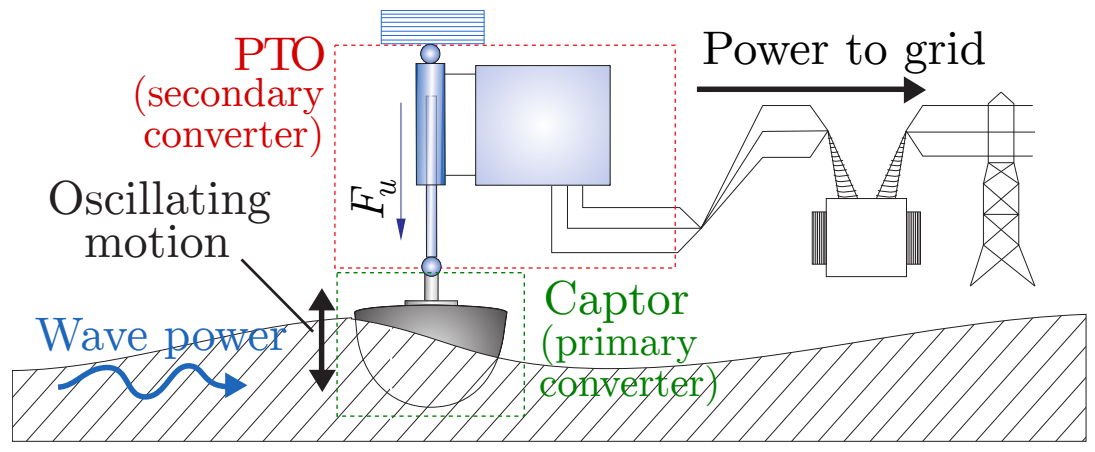

Figure 1: Schematic diagram of a wave energy converter of the point-absorber type

The PTO can be used as an actuator to adjust the natural response of the captor to waves, in order to maximize the extracted energy. The ideal conditions for optimal energy absorption have been studied in [1, showing that an energy maximizing controller requires future knowledge of the wave excitation force $F_{e x}$, that is, the force exerted by the incoming wave on the captor. Among the many different approaches to hydrodynamic control of WECs, see [2] for a thorough review, latching control [3], 4], declutching control [5], and model predictive control (MPC) 6] are examples of strategies relying, directly or indirectly, on this knowledge. In the MPC context, for instance, the complete control scheme must include an online algorithm to compute future values of the wave excitation force over the prediction horizon, as shown in Figure 2.

Notice that, while it is relatively straightforward to measure excitation force using a dedicated experiment and a well-positioned force sensor [7, only indirect measurements or estimations are possible during normal WEC operation. Two experimentally-validated methods for wave force estimation from available measurements are described in [7. Assuming that local wave elevation measurements are possible during WEC operation, another, less direct, approach could consist of computing future values of the wave excitation force from wave elevation predictions, though this would require an inconvenient increase of the prediction horizon, to cope with the non-causal nature of the impulse function 


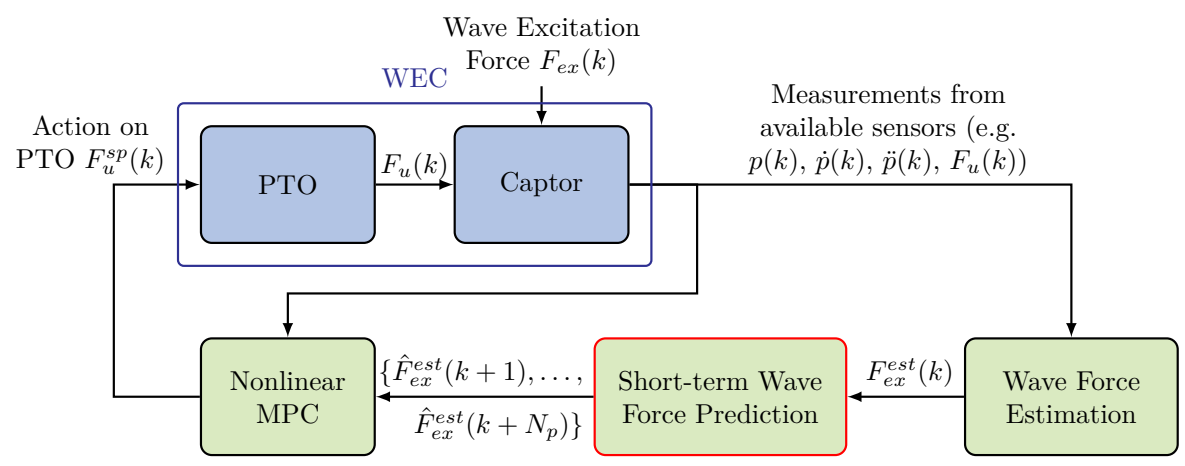

Figure 2: Wave excitation force prediction in the context of MPC

relating wave elevation to wave excitation force. Indeed, for its important role in the optimization of WEC energy yield, short-term wave forecasting, with a particular focus on wave elevation, has drawn a lot of attention in the hydrodynamic control community.

A first possible approach to perform short-term wave forecasting is spatial prediction, using up-wave measurements from sensors installed around the location of a WEC [8], 9], 10]. The method is reported to forecast quite long prediction horizons with a good performance [11]. However the forecasting model can become very complex, since the wave propagation nonlinearities or/and the possible multi-directionality of waves have to be taken into account [12].

A second approach, that has become popular in the last years because of its simplicity, is to use past time series of local measurements or estimates, at the float position. In [13, using real wave elevation data, Fusco and Ringwood show that a relatively simple linear auto-regressive (AR) model can perform quite well, provided that the high-frequency content is filtered out from the time series data. To avoid introducing a phase lag, the use of a non-causal zero-phase filter is advocated. The solution is based on a batch-processing approach, which also includes a computationally-expensive nonlinear least squares problem to be solved and a spectral analysis to be performed in order to compute an optimal sampling period for all the computations. It is worth noticing that considering 
a more complex model structure in this context, namely an auto regressive moving average (ARMA) model instead of an AR model, does not seem to bring any particular benefit [14]. In [15], an iterative, more easily implementable approach is proposed, based on a bank of least squares estimators. However, as it will be shown later, it is implicitly assumed that the sea state is constant. Furthermore, as noticed in [15], the prediction performance degrades as quickly as the prediction horizon increases.

Two novel solutions for short-term wave forecasting are proposed in this paper. They are also based on past time series of local WEC measurements or estimates. Implementation aspects such as computational complexity and accuracy are investigated. Their performance is assessed using wave excitation force time series, obtained from data collected in the wave basin of Aalborg University, on a lab-scale wave energy converter prototype.

Three main features of the proposed solutions, built around AR-model estimation, are:

- It is shown that, for the first method, the multi-step ahead error criterion adopted in [13] is a particular case of our criterion.

- The first method is based on the extended Kalman filter. Hence the algorithm is recursive and easy to implement.

- To improve the performance, overcoming the error accumulation problem that comes with the first method, an alternative approach is proposed. It builds an independent model for each horizon, using an adaptive Kalman filter. It is also shown that the approach in [15] is a limiting case of ours, when the sea state is assumed to be constant.

The paper is organized as follows. The problem is formulated in Section 2 together with a review from the literature. Then the multi-step error minimization approach with extended Kalman filter is proposed in Section 3, while in Section 4, an adaptive Kalman filtering approach is considered. In Section 5 . the available data as well as the prediction results are presented. In Section 6 
the computation of a forecasting interval is considered. Some conclusions are drawn in Section 7 .

\section{PROBLEM FORMULATION}

Given available estimates $\left\{\hat{F}_{e x}(l)\right\}$ of the wave excitation force taken at discrete time instants $l=0,1, \ldots, k$, where $k$ is the current time, our objective is to predict the wave excitation force at time $k+1, k+2, \ldots, k+N_{p}$, where $N_{p}$ is the prediction horizon. For this purpose, some prediction methods in 85 the literature are first reviewed. These methods will be compared to the new approaches developed in the paper. In the following, for simplicity denote $y(k)=$ $\hat{F}_{e x}(k)$.

\subsection{Decomposition based approach}

This approach is based on the assumption that $y(k)$ may be regarded as the

90 sum of several sinusoidal waves of different frequencies, amplitudes and phases,

$$
y(k)=\sum_{j=1}^{m} A_{j} \sin \left(\omega_{j} k+\phi_{j}\right)
$$

where $m$ is the total number of components, $A_{j}, \omega_{j}$ and $\phi_{j}$ are the amplitude, the angular wave frequency and the phase angle of the jth component, respectively [16]. Note that in the model (1) the frequencies $\omega_{j}$ are known and fixed, while $A_{j}$ and $\phi_{j}$ are unknown. The parameters $A_{j}$ and $\phi_{j}$ can be estimated through 95 least squares or Kalman filter procedures and can be used to forecast the future wave excitation force [13].

The main advantage of the model 11 is its direct physical meaning. However the constant frequencies assumption is rather restrictive and unrealistic, since it is well known that the wave excitation force spectrum is time-varying [17. Consequently, it is not reliable to use the model (1) to predict the future wave excitation force. 


\subsection{Sinusoidal extrapolation based approach}

The idea is to model $y(k)$ as a single sinusoidal signal with a time-varying frequency, amplitude and phase,

$$
y(k)=A(k) \sin (\omega(k) k+\phi(k))
$$

105

where $A(k), \omega(k)$ and $\phi(k)$ are unknown.

Evidently, the model (2) is nonlinear in $A(k), \omega(k)$ and $\phi(k)$. As a consequence, a linear recursive estimator cannot be directly applied. It is possible, however, to use a truncated Taylor expansion of (2), and then an extended Kalman filter (EKF) to estimate the values of $A(k), \omega(k)$ and $\phi(k)[13$.

A direct physical meaning is also an advantage of model (2). However, it is clear that using one sinusoid to describe a wave is only effective for very narrowbanded wave systems. In addition, the extension to a model with multiple time-varying frequencies is not as straightforward as it may seem.

\subsection{Auto-regressive model based approach}

The AR model is based on the assumption that $y(k)$ at time $k$ is a linear function of its past observations $y(k-1), y(k-2), \ldots, y(k-p)$, i.e.

$$
y(k)=a_{1} y(k-1)+a_{2} y(k-2)+\ldots+a_{p} y(k-p)+w(k)
$$

where $\left(a_{1}, a_{2}, \ldots, a_{p}\right)$ are the parameters, $p$ is the order of the model, and $w(k)$ is a zero-mean white noise disturbance.

Assume that, at time $k$, the coefficients $a_{j}, \forall j=1,2, \ldots, p$ are already calculated. Note that $a_{j}, \forall j=1,2, \ldots, p$ might be time-varying, i.e. $a_{j}=a_{j}(k)$ might also be functions of time. It is well known [18] that the best prediction of $\hat{y}(k+h \mid k)$ at time $k$ can be calculated as,

$$
\hat{y}(k+h \mid k)=a_{1}(k) \hat{y}(k+h-1 \mid k)+a_{2}(k) \hat{y}(k+h-2 \mid k)+\ldots+a_{p}(k) \hat{y}(k+h-p \mid k)
$$

where $\hat{y}(k+h-j \mid k)=y(k+h-j)$ if $k+h-j \leq k$ or equivalently, if $h-j \leq$ 0 . It is shown in [13] that the AR model (3) with only complex-conjugate 
Rewrite $\sqrt{3}$ in a compact vector form as

$$
y(k)=x_{a r}(k-1)^{T} \mathbf{a}+w(k)
$$

where

$$
\left\{\begin{array}{l}
x_{a r}(k-1)=\left[\begin{array}{llll}
y(k-1) & y(k-2) & \ldots & y(k-p)
\end{array}\right]^{T}, \\
\mathbf{a}=\left[\begin{array}{llll}
a_{1} & a_{2} & \ldots & a_{p}
\end{array}\right]^{T}
\end{array}\right.
$$

The simplest way to estimate $a_{j}, j=1,2, \ldots, p$ at time $k$, consists of minimizing

$$
\min _{a_{1}, a_{2}, \ldots, a_{p}} \sum_{l=p+1}^{k}(y(l)-\hat{y}(l \mid l-1))^{2}
$$

It is well known [19] that the optimal solution $\mathbf{a}^{*}$ to 6 can be obtained analytically.

Theoretically, if the model structure $(3)$ exactly matches the structure of the actual system, and the signal to noise ratio is high, then the model obtained using (6) is the best linear unbiased estimator, which also provides optimal multi-step ahead predictions. However, Fourier analysis reveals that real wave excitation force estimates are generally quite noisy. In addition, it is not possible to know the exact model structure of the real system to be identified. Consequently, it is not reliable to use the cost function (6) to produce accurate predictions over an entire forecasting horizon.

One way to improve the accuracy given by model (3) in forecasting is to consider the multi-step ahead error minimization, referred to as long-range predictive identification (LRPI) 20, [13,

$$
\min _{a_{1}, a_{2}, \ldots, a_{p}} \sum_{l=p+M+1}^{k} \sum_{j=1}^{M}(y(l)-\hat{y}(l \mid l-j))^{2}
$$

where $M$ is the forecasting horizon over which the AR model is to be optimized.

Parameters estimated from objective functions based on multi-step ahead predictors, generally result in better models for wave forecasting. In addition to 
the time domain interpretation, Wahlberg and Ljung [21] showed that the use of multi-step ahead error minimization method amounts to emphasizing the accuracy of low-frequency dynamics more in distributing the bias, compared to the one-step-ahead error minimization, which tends to put higher emphasis on the high frequency behavior.

Unfortunately, the optimization problem based on the multi-step ahead error minimization criterion (7) is nonlinear. Hence, in general, no analytical solution $a_{j}^{*}$ can be found. One may, however, use the Gauss-Newton algorithm to obtain a local optimal solution as indicated in [13].

\section{EXTENDED KALMAN FILTERING APPROACH}

The Gauss-Newton based solution in Section 2.3 is a batch - processing approach and in a non-recursive form, which might be difficult to realize in practice. The solution is ill-adapted for on-line identification because of its computational and storage costs. In addition, until a new optimal solution $a_{j}^{*}$ is found, it is implicitly assumed that the coefficients $a_{j}^{*}$ are constant. This assumption, as it will be shown later, is rather restrictive.

The main contribution of this section is to propose a new algorithm for the multi-step ahead error minimization method. The approach is based on the EKF procedure, and hence is recursive. To the best of the authors' knowledge, this is the first time that the EKF is applied to the multi-step ahead error minimization method. The sea state time-varying nature is handled via a process noise in the state equation, as it will be shown in the next section.

\subsection{State space equation for the filter}

Since the sea state varies with time, but the variation is slow, it can be assumed that, $\forall j=1,2, \ldots, p$,

$$
a_{j}(k+1)=a_{j}(k)+\eta_{j}(k)
$$

where $\eta_{j}(k)$ is used to describe the variation of $a_{j}(k)$. 
Remark 1: It is worth noticing that allowing $a_{j}(k)$ to be time-varying is also a way to compensate the fact that the AR model (3) is only an approximation of the real wave excitation force model.

Equation 8 can be rewritten in a compact vector form as,

$$
\mathbf{a}(k+1)=\mathbf{a}(k)+\eta(k)
$$

where

$$
\left\{\begin{array}{l}
\mathbf{a}(k)=\left[\begin{array}{llll}
a_{1}(k) & a_{2}(k) & \ldots & a_{p}(k)
\end{array}\right]^{T}, \\
\eta(k)=\left[\begin{array}{llll}
\eta_{1}(k) & \eta_{2}(k) & \ldots & \eta_{p}(k)
\end{array}\right]^{T}
\end{array}\right.
$$

At time $k$, for the multi-step ahead error minimization method, one would like to have:

- A small one-step ahead prediction error

$$
\epsilon_{1}(k)=y(k)-\hat{y}(k \mid k-1)
$$

where

$$
\hat{y}(k \mid k-1)=x_{a r}(k-1)^{T} \mathbf{a}(k) .
$$

- A small two-step ahead prediction error

$$
\epsilon_{2}(k)=y(k)-\hat{y}(k \mid k-2)
$$

where $\hat{y}(k \mid k-2)$ is the wave prediction at time $k$ using $y(k-2), y(k-3), \ldots$ The value of $\hat{y}(k \mid k-2)$ can be calculated iteratively via $\hat{y}(k-1 \mid k-2)$ as

$$
\hat{y}(k \mid k-2)=a_{1}(k) \hat{y}(k-1 \mid k-2)+a_{2}(k) y(k-2)+\ldots+a_{p}(k) y(k-p)
$$

where

$$
\hat{y}(k-1 \mid k-2)=a_{1}(k) y(k-2)+a_{2}(k) y(k-3)+\ldots+a_{p}(k) y(k-p-1)
$$

Hence

$$
\begin{aligned}
\hat{y}(k \mid k-2)= & \left(a_{1}(k)^{2}+a_{2}(k)\right) y(k-2)+\left(a_{1}(k) a_{2}(k)+a_{3}(k)\right) y(k-3) \\
& +\ldots+a_{1}(k) a_{p}(k) y(k-p-1)
\end{aligned}
$$


- A small $l$-step ahead prediction error, $\forall l=3,4, \ldots, M$

$$
\epsilon_{l}(k)=y(k)-\hat{y}(k \mid k-l)
$$

where $\hat{y}(k \mid k-l)$ is the prediction at time $k$ using $y(k-l), y(k-l-1), \ldots$ The value of $\hat{y}(k \mid k-l)$ can be calculated in a similar way as for $\hat{y}(k \mid k-2)$. Recall that $M$ is the forecasting horizon over which the AR model is to be optimized.

Combining (10, 11], 13), one obtains,

$$
\left\{\begin{array}{l}
y(k)=\hat{y}(k \mid k-1)+\epsilon_{1}(k), \\
y(k)=\hat{y}(k \mid k-2)+\epsilon_{2}(k), \\
\vdots \\
y(k)=\hat{y}(k \mid k-M)+\epsilon_{M}(k)
\end{array}\right.
$$

Equation (14) is considered as an output equation for the EKF. The residuals $\epsilon_{j}(k), \forall j=1,2, \ldots, M$ are assumed to be a noise, which also represents the measurement disturbance.

Together with (8), one gets the following state space equation,

$$
\left\{\begin{array}{c}
\mathbf{a}(k+1)=\mathbf{a}(k)+\eta(k), \\
{\left[\begin{array}{c}
y(k) \\
y(k) \\
\vdots \\
y(k)
\end{array}\right]=\left[\begin{array}{c}
\hat{y}(k \mid k-1) \\
\hat{y}(k \mid k-2) \\
\vdots \\
\hat{y}(k \mid k-M)
\end{array}\right]+\epsilon(k)}
\end{array}\right.
$$

where $\epsilon(k)=\left[\epsilon_{1}(k) \epsilon_{2}(k) \ldots \epsilon_{M}(k)\right]^{T}$.

The state equation of 15 is linear with respect to (w.r.t) the state or the parameters $a_{j}, j=1,2, \ldots, p$. However, using 12, it is clear that the output equation of 15$]$ is nonlinear w.r.t. $a_{j}$. In this paper, to estimate $\mathbf{a}(k)$, the EKF method is applied due to its simplicity, optimality, tractability and robustness. 


\subsection{Extended Kalman filter}

In essence, for system (15), the EKF filter provides a solution to the following minimization problem

$$
\min _{\mathbf{a}(k)} J_{a}(k)
$$

with a cost function defined as

$$
\begin{aligned}
J_{a}(k)=(\mathbf{a}(0)-\mathbf{a}(0 \mid 0))^{T} P_{0}^{-1}(\mathbf{a}(0)-\mathbf{a}(0 \mid 0))+ \\
\quad+\sum_{l=1}^{k}\left(\eta(l-1)^{T} Q^{-1} \eta(l-1)+\epsilon(l)^{T} R^{-1} \epsilon(l)\right)
\end{aligned}
$$

where $\mathbf{a}(0 \mid 0)$ is the mean value of $\mathbf{a}(0), P_{0}, Q, R$ are weighting matrices. Recall that $\eta(k)=\mathbf{a}(k+1)-\mathbf{a}(k)$, and

$$
\epsilon(k)=\left[\begin{array}{c}
y(k) \\
y(k) \\
\vdots \\
y(k)
\end{array}\right]-\left[\begin{array}{c}
\hat{y}(k \mid k-1) \\
\hat{y}(k \mid k-2) \\
\vdots \\
\hat{y}(k \mid k-M)
\end{array}\right]
$$

Consider the following limiting case, where

- No information about initial state $\mathbf{a}(0)$ is available. Then one should choose a large $P_{0}$. Therefore $(\mathbf{a}(0)-\mathbf{a}(0 \mid 0))^{T} P_{0}^{-1}(\mathbf{a}(0)-\mathbf{a}(0 \mid 0))$ can be neglected in the objective function (17).

- The sea state is time-invariant. Hence, $\forall k$,

$$
\mathbf{a}(k+1)=\mathbf{a}(k)
$$

and $\eta(k)=0$.

In this case, the cost function 17 can be rewritten as,

$$
J_{a}(k)=\min _{\mathbf{a}} \sum_{l=1}^{k} \epsilon(l)^{T} R^{-1} \epsilon(l)
$$

15 If $R$ is the identity matrix, and by re-indexing $l$, one obtains

$$
J_{a}(k)=\min _{\mathbf{a}} \sum_{l=p+M+1}^{k} \sum_{j=1}^{M}(y(l)-\hat{y}(l \mid l-j))^{2}
$$


Thus one obtains the cost function (7) of LRPI. Hence (7) is a particular case of (17), when the sea state is time invariant and the initial parameter $\mathbf{a}(0)$ is completely unknown.

Remark 2: Note that the multi-step ahead error minimization cost (7) can be used to obtain information about $\mathbf{a}(0)$. However, a solution of a nonlinear least squares problems is required. In this work, the matrix $P_{0}$ has been set large. This implies no information about $\mathbf{a}(0)$ is available.

Remark 3: Recall that an estimator [7] is used to estimate the wave excitation force $y(k)$ in this work. Consequently, the measurement noise matrix $R$ for the EKF is known. There are several methods in the literature to estimate the process noise matrix $Q$ such as maximum like-hood estimation [22, correlation method [23], 24], etc. However due to the limited time available for the tests, the trial and error method is used to tune $Q$. The basic idea is to first try to find the main diagonal, and then the first and the second sub-diagonal elements of $Q$. The other sub-diagonal elements of $Q$ are set to be zero in this work.

The following notations are adopted,

- $\hat{\mathbf{a}}(k \mid k-1)$ is the estimate of $\mathbf{a}(k)$ given measurements from time $k-1$, i.e. $y(k-1), y(k-2), \ldots$,

- $\hat{\mathbf{a}}(k \mid k)$ is the estimate of $\mathbf{a}(k)$ given measurements from time $k$, i.e. $y(k), y(k-$ 235 $1), \ldots$,

- $P(k \mid k-1)$ is the covariance matrix of $\mathbf{a}(k)$ given $y(k-1), y(k-2), \ldots$

- $P(k \mid k)$ is the covariance matrix of $\mathbf{a}(k)$ given $y(k), y(k-1), \ldots$,

Then the EKF procedure is summarized as follows,

- Time update,

$$
\begin{cases}\hat{\mathbf{a}}(k \mid k-1) & =\hat{\mathbf{a}}(k-1 \mid k-1), \\ P(k \mid k-1) & =P(k-1 \mid k-1)+Q\end{cases}
$$


- Measurement update,

$$
\left\{\begin{aligned}
& K(k)=P(k \mid k-1) H(k)\left(H(k)^{T} P(k \mid k-1) H(k)+R\right)^{-1} \\
& \hat{\mathbf{a}}(k \mid k)=\hat{\mathbf{a}}(k \mid k-1)+K(k)\left(\left[\begin{array}{c}
y(k) \\
y(k) \\
\vdots \\
y(k)
\end{array}\right]-\left[\begin{array}{c}
\hat{y}(k \mid k-1) \\
\hat{y}(k \mid k-2) \\
\vdots \\
\hat{y}(k \mid k-M)
\end{array}\right]\right) \\
& P(k \mid k)=(I-K(k) H(k)) P(k \mid k-1)
\end{aligned}\right.
$$

with

$$
H(k)=\left.\frac{d h(k)}{d \mathbf{a}}\right|_{\mathbf{a}=\hat{\mathbf{a}}(k \mid k-1)}
$$

where $I$ is the identity matrix of appropriate dimension, and

$$
h(k)=\left[\begin{array}{c}
\hat{y}(k \mid k-1) \\
\hat{y}(k \mid k-2) \\
\vdots \\
\hat{y}(k \mid k-M)
\end{array}\right]
$$

Once the optimal parameter $\hat{\mathbf{a}}(k \mid k)$ is found, it can be used to forecast the wave excitation force as follows,

Algorithm 1: Wave force forecasting based on parameters estimated by the EKF filter

1. Input: Wave estimates $y(k), y(k-1), \ldots$, Estimated parameters $\hat{\mathbf{a}}(k \mid k)$, Forecasting horizon $N_{p}$

2. Output: $\hat{y}(k+1 \mid k), \hat{y}(k+2 \mid k), \ldots, \hat{y}\left(k+N_{p} \mid k\right)$

(i) Initialization $s=1$ and

$$
x=[y(k) y(k-1) \ldots y(k-p+1)]^{T}
$$


(ii) Calculate the predictions $\hat{y}(k+s \mid k)$ as,

$$
\left\{\begin{array}{l}
y_{f}=x^{T} \hat{\mathbf{a}}(k \mid k), \\
\hat{y}(k+s \mid k)=y_{f}, \\
x=\left[y_{f} x(1: p-1)^{T}\right]^{T}, \\
s=s+1
\end{array}\right.
$$

(iii) If $s \leq N_{p}$, then go to Step (ii), otherwise Stop.

Algorithm 1 is schematically presented in Figure 3 It is worth noticing that $\hat{y}(k+j \mid k)$ is used to predict $\hat{y}(k+j+1 \mid k)$. Hence Algorithm 1 can be classified as an iterative procedure. Since only one model is required, significant computational time can be saved, especially when the forecast horizon is large.

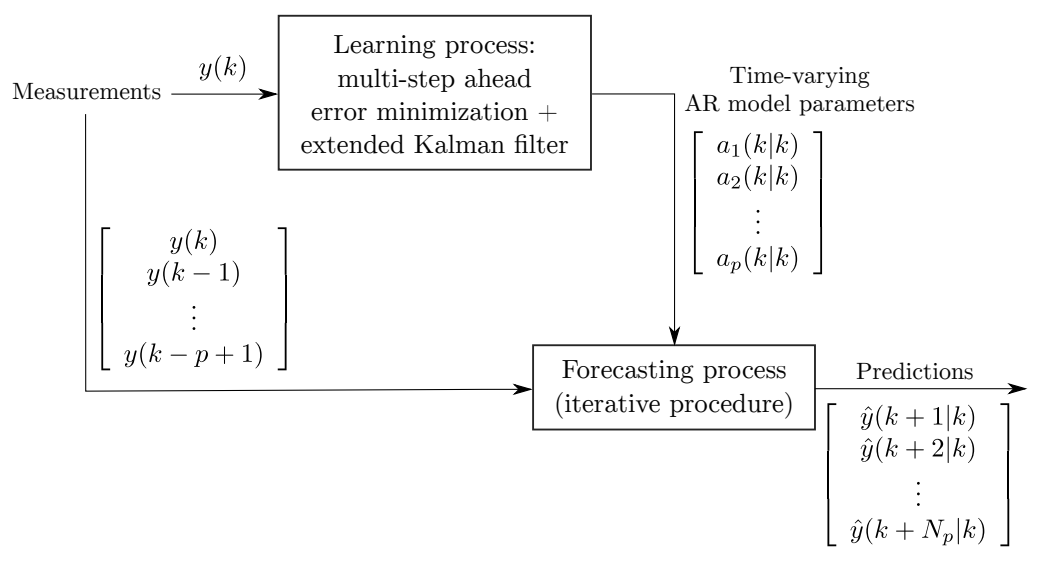

Figure 3: Synoptic of the wave excitation force prediction method using EKF filter.

\section{ADAPTIVE KALMAN FILTERING APPROACH}

As discussed in Section 3 low computational complexity is the main advantage of algorithm 1. However, since the predicted values from the past are used to predict the future, it can be shown that the approach suffers from an error 
accumulation problem, i.e. errors committed in the past are propagated into future predictions.

For the short-term wave excitation force forecasting, an alternative approach, that does not suffer from the error accumulation problem, is proposed in this Section. The main idea is to use an optimal $h$-step ahead prediction model for each $h=1,2, \ldots, N_{p}$, where $N_{p}$ is the prediction horizon. Then all the $N_{p}$ models are simultaneously used for predictions.

The new method is based on the assumption that the wave excitation force $y(k+h)$ at time $k+h$ is a linear function of its past estimates $y(k), y(k-$ $1), \ldots, y(k-p+1)$, i.e.

$$
y(k+h)=\sum_{j=1}^{p} a_{j, h}(k) y(k-j+1)+\epsilon(k+h)
$$

where $h=1,2, \ldots, N_{p}$ is the forecasting horizon, $p$ is the order of the model. Note that $p$ can be different for different $h . \epsilon(k+h)$ is a zero mean white noise. $\mathbf{a}_{h}(k)=\left[\begin{array}{llll}a_{1, h}(k) & a_{2, h}(k) & \ldots & a_{p, h}(k)\end{array}\right]^{T}$ are the parameters to be estimate.

At time $k$, it is well known [18] that the best prediction of the future wave excitation force $\hat{y}(k+h \mid k)$ is

$$
\hat{y}(k+h \mid k)=\sum_{j=1}^{p} a_{j, h}(k) y(k-j+1)
$$

Clearly, $\mathbf{a}_{h}(k)$ need to be estimated in order to forecast $\hat{y}(k+h \mid k)$. One of the most common ways to do this is to assume that

$$
\mathbf{a}_{h}(k+1)=\mathbf{a}_{h}(k)
$$

and then to solve the following least squares optimization problem [15]

$$
\min _{\mathbf{a}_{h}} \sum_{l=p+h+1}^{k}(y(l)-\hat{y}(l \mid l-h))^{2}
$$

which one will refer to as multi-model long range prediction identification (MMLRPI), or multi-model multi-step ahead error minimization. It is well known [15], 25] that the solution to (24) can be found analytically using the least squares method. 
It is worth noticing that equation 23 does not take into account the sea state time-varying nature. A more realistic solution to find $\mathbf{a}_{h}(k)$, is to use the weighted least squares method, resulting in the following cost function,

$$
\min _{\mathbf{a}_{h}} \sum_{l=p+h+1}^{k} \lambda^{k-l}(y(l)-\hat{y}(l \mid l-h))^{2}
$$

where $\lambda \in\left(\begin{array}{ll}0 & 1\end{array}\right]$ is the forgetting factor. However, it is well known [26] that, when the measurements do not give any new information to the system, one might have the blow-up phenomenon with the weighted least squares method. of sea state is taken into account. For this purpose, rewrite $(21)$ as,

$$
y(k)=\sum_{j=1}^{p} a_{j, h}(k-h) y(k-h-j+1)+\epsilon(k)
$$

Since the sea state is time-varying, but the variation is slow, it can be assumed that, $\forall j=1,2, \ldots, p$,

$$
a_{j, h}(k+1)=a_{j, h}(k)+\eta_{j}(k)
$$

where $\eta_{j}(k), \forall j=1,2, \ldots, p$, are the noises. Denote

$$
\eta(k)=\left[\eta_{1}(k) \eta_{2}(k) \ldots \eta_{p}(k)\right]^{T}
$$

Using (27), one has,

$$
\begin{aligned}
\mathbf{a}_{h}(k) & =\mathbf{a}_{h}(k-1)+\eta(k-1) \\
& =\mathbf{a}_{h}(k-2)+\eta(k-2)+\eta(k-1) \\
& =\ldots \\
& =\mathbf{a}_{h}(k-h)+\sum_{v=1}^{h} \eta(k-v)
\end{aligned}
$$

Hence

$$
\mathbf{a}_{h}(k-h)=\mathbf{a}_{h}(k)-\sum_{v=1}^{h} \eta(k-v)
$$

Equation (26) can be rewritten as

$$
y(k)=x_{h}(k)^{T} \mathbf{a}_{h}(k)+\mu(k)
$$


where

$$
\left\{\begin{array}{l}
x_{h}(k)=[y(k-h) y(k-h-1) \ldots y(k-h-p+1)]^{T}, \\
\mu(k)=-x_{h}(k)^{T} \sum_{v=1}^{h} \eta(k-v)+\epsilon(k)
\end{array}\right.
$$

Combining (27), (29), the following state space equation is obtained

$$
\begin{cases}\mathbf{a}_{h}(k+1) & =\mathbf{a}_{h}(k)+\eta(k), \\ y(k) & =\sum_{j=1}^{p} a_{j, h}(k) y(k-h-j+1)+\mu(k)\end{cases}
$$

Evidently, $\mathbf{a}_{h}(k)$ enters linearly in equation 30 . One way to estimate optimally and recursively the unknown state vector $\mathbf{a}_{h}(k)$ is to apply the linear Kalman filter (LKF) algorithm 27. In essence, the LKF provides the solution of the optimization problem

$$
\min _{\mathbf{a}_{h}(k)} J_{a}(k)
$$

with

$$
\begin{aligned}
J_{a}(k)=\left(\mathbf{a}_{h}(0)-\mathbf{a}_{h}(0 \mid 0)\right)^{T} P_{0}^{-1}\left(\mathbf{a}_{h}(0)-\mathbf{a}_{h}(0 \mid 0)\right)+ \\
\quad+\sum_{l=1}^{k}\left(\eta(l-1)^{T} Q_{h}^{-1} \eta(l-1)+\mu(l)^{T} R_{h}^{-1} \mu(l)\right)
\end{aligned}
$$

where $P_{0}, Q_{h}, R_{h}$ are weighting matrices, $\mathbf{a}_{h}(0 \mid 0)$ is the mean value of the initial state $\mathbf{a}_{h}(0 \mid 0)$. Recall that

$$
\left\{\begin{array}{l}
\eta(k)=\mathbf{a}_{h}(k+1)-\mathbf{a}_{h}(k) \\
\mu(k)=y(k)-\sum_{j=1}^{p} a_{j, h}(k) y(k-h-j+1)
\end{array}\right.
$$

Consider now the following limit case, where

- No information about initial state $\mathbf{a}(0)$ is available. Hence $P_{0}$ should be chosen very large. As a consequence $\left(\mathbf{a}_{h}(0)-\mathbf{a}_{h}(0 \mid 0)\right)^{T} P_{0}^{-1}\left(\mathbf{a}_{h}(0)-\right.$ $\left.\mathbf{a}_{h}(0 \mid 0)\right)$ is a negligible term in the cost function (31).

- The sea state is time-invariant. Hence, $\forall k$,

$$
\mathbf{a}(k+1)=\mathbf{a}(k)
$$

and $\eta(k)=0$. 
In this case, the cost function 31 can be rewritten as,

$$
J_{a}(k)=\min _{\mathbf{a}} \sum_{l=1}^{k} \mu(l)^{T} R_{h}^{-1} \mu(l)
$$

If $R_{h}$ is the identity matrix, and by re-indexing $l$, one obtains

$$
J_{a}(k)=\min _{\mathbf{a}_{h}} \sum_{l=p+h+1}^{l}(y(l)-\hat{y}(l \mid l-h))^{2}
$$

315 Thus the cost function (24) of MM-LRPI is obtained. Hence (24) is a particular case of (27), when the sea state is time invariant and the initial parameter $\mathbf{a}(0)$ is completely unknown.

Remark 4: With the appropriate choice of forgetting factor, the method in [15] can also cope with a time-varying sea state. However, beside the fact that the weighted least squares method might lead to the blow-up phenomenon, the Kalman filter based method has more tuning parameters, i.e., more degrees of freedom than the weighted least squares method. Hence the Kalman filter based method is a better choice than the weighted least squares based method.

Using the same notation as in Section 3 for $\hat{\mathbf{a}}_{h}(k \mid k-1), \hat{\mathbf{a}}_{h}(k \mid k), P_{h}(k \mid k-1)$ and $P_{h}(k \mid k)$, the LKF algorithm is summarized as follows

- Time update

$$
\left\{\begin{array}{l}
\hat{\mathbf{a}}_{h}(k \mid k-1)=\hat{\mathbf{a}}_{h}(k-1 \mid k-1), \\
P_{h}(k \mid k-1)=P_{h}(k-1 \mid k-1)+Q_{h}
\end{array}\right.
$$

- Measurement update

$$
\left\{\begin{array}{l}
K_{h}(k)=P_{h}(k \mid k-1) x_{h}(k)\left(x_{h}(k)^{T} P_{h}(k \mid k-1) x_{h}(k)+R_{h}\right)^{-1} \\
\hat{\mathbf{a}}_{h}(k \mid k)=\hat{\mathbf{a}}_{h}(k \mid k-1)+K(k)\left(y(k)-x_{h}(k)^{T} \hat{\mathbf{a}}_{h}(k \mid k-1)\right), \\
P_{h}(k \mid k)=\left(I-K(k) x_{h}(k)\right) P_{h}(k \mid k-1)
\end{array}\right.
$$

where

$$
x_{h}(k)=[y(k-h) y(k-h-1) \ldots y(k-h-p+1)]^{T},
$$

Once the optimal parameter $\mathbf{a}_{h}(k \mid k)$ is calculated, it can be used in producing the forecasting horizon $\hat{y}(k+h \mid k)$ as follows 
Algorithm 2: Wave force forecasting based on parameters estimated by the AKF filters

1. Input: Wave estimates $y(k), y(k-1), \ldots$, Prediction horizon $N_{p}$, Covariance matrices $Q_{h}, R_{h}$, Estimations $\hat{\mathbf{a}}_{h}(k-1 \mid k-1), P_{h}(k-1, k-1)$,

335 $\forall h=1,2, \ldots, N_{p}$

2. Output: Prediction $\hat{y}(k+h \mid k), \forall h=1,2, \ldots, N_{p}$

(i) Initialization: $h=1$

(ii) Calculate the vector,

$$
x_{a r}(k)=\left[\begin{array}{llll}
y(k) & y(k-1) & \ldots & y(k-p+1)
\end{array}\right]^{T}
$$

(iii) Apply the LKF (32), 33, 34 to obtain $\hat{\mathbf{a}}_{h}(k \mid k), P_{h}(k, k)$

(iv) The prediction $\hat{y}(k+h \mid k)$ is computed as,

$$
\hat{y}(k+h \mid k)=x_{a r}(k)^{T} \hat{\mathbf{a}}_{h}(k \mid k)
$$

(v) Set $h:=h+1$

(vi) If $h \leq N_{p}$, then go to Step (iii). Otherwise Stop.

Algorithm 2 is schematically presented in Figure 4. Note that $N_{p}$ linear Kalman filters are used to obtain $\hat{y}(k+1 \mid k), \hat{y}(k+2 \mid k), \ldots, \hat{y}\left(k+N_{p} \mid k\right)$. Consequently, this approach involves a heavier computational burden than iterative forecasting.

\section{EXPERIMENTAL RESULTS}

\subsection{Available data}

The wave data utilized for this study were collected in a wave basin of Aalborg University ( $15 \mathrm{~m}$ long, $8 \mathrm{~m}$ wide and $0.7 \mathrm{~m}$ deep), in the context of an experimental assessment of the performance of a nonlinear MPC strategy applied to a WEC prototype [28].

The WEC under consideration (shown in Figure 5 is a $1: 20$ scale laboratory prototype of the well-known Wavestar machine installed near Hanstholm 


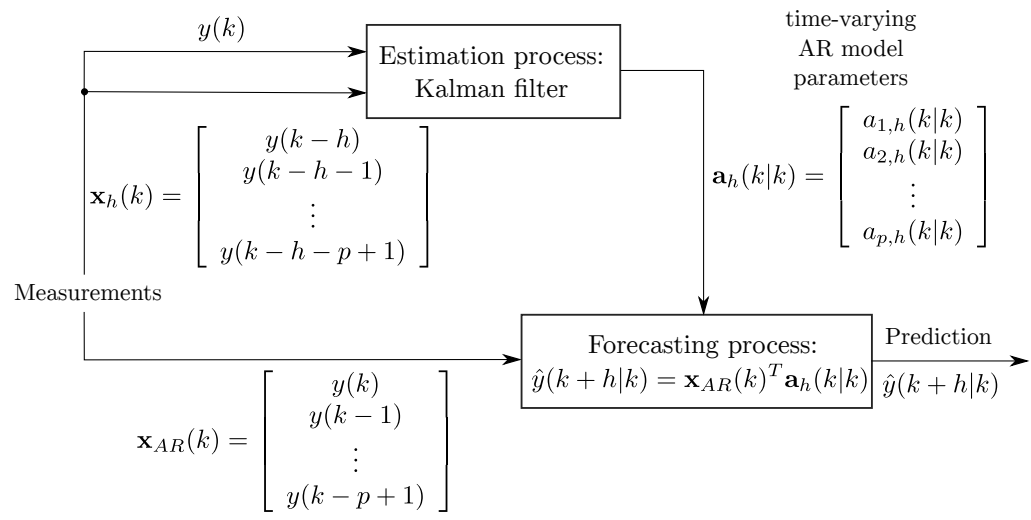

Figure 4: Synoptic of the wave excitation force prediction method using AKF filter.

355

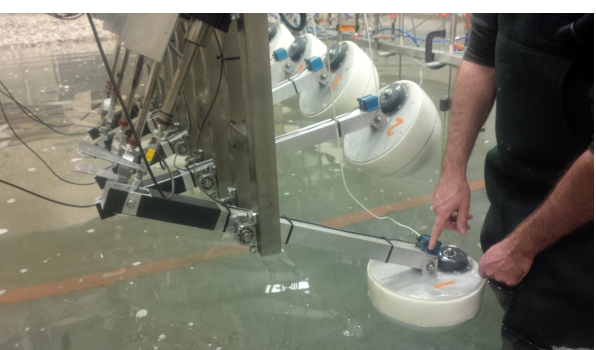

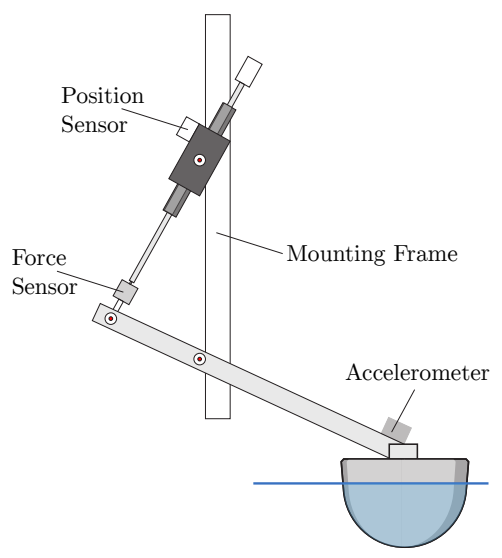

Figure 5: Experimental set-up at the wave basin of Aalborg University

A linear motor is used to emulate the action of the PTO. An accelerometer and a position sensor provide measurements of the float motion, while a load cell provides a measurement of the force applied by the linear motor. A rapid control prototyping architecture, based on the Matlab xPCTarget toolbox, allows to run controllers developped in Simulink in real-time (up to $1 \mathrm{kHz}$ ) on a target PC 
connected to the WEC I/O board. In Simulink, all the linear measurements are transparently converted into the angular reference frame corresponding to the single degree of freedom of the WEC (rotation around the hinge point). Float angular velocity is estimated from acceleration and position, using a Kalman filter.

The MPC controller validated in the experimental campaign has the structure represented in Figure 2, except that position, velocity and acceleration are angular, and moments replace forces.

The data considered for the present analysis are time series of wave moment estimates (the output of the "Wave Force Estimation" block in Figure 2, logged during closed-loop experiments where MPC is used to maximize WEC electric energy production, under the action of different irregular waves. A set of four different irregular waves, each generated from a different wave spectrum, is considered. The spectra are representative of the most common sea state conditions in Hanstholm, adapted in scale to the WEC prototype in the basin, via the Froude similarity laws (see Table 1). The waves are unidirectional, two dimensional, long-crested waves, and are generated by a wave maker whose paddles are driven by a total of 15 hydraulic pistons moving in the horizontal direction.

\begin{tabular}{|l|c|c|c|}
\hline & Peak period & $\begin{array}{c}\text { Significant } \\
\text { wave height }\end{array}$ & $\begin{array}{c}\text { Probability of } \\
\text { occurrence in Hanstholm }\end{array}$ \\
\hline Sea state 1 & $0.7407 \mathrm{~s}$ & $0.0620 \mathrm{~m}$ & $30 \%$ \\
\hline Sea state 2 & $0.9259 \mathrm{~s}$ & $0.0920 \mathrm{~m}$ & $45 \%$ \\
\hline Sea state 3 & $1.1111 \mathrm{~s}$ & $0.1220 \mathrm{~m}$ & $10 \%$ \\
\hline Sea state 4 & $1.4815 \mathrm{~s}$ & $0.1800 \mathrm{~m}$ & Extreme operation \\
\hline
\end{tabular}

Table 1: Sea state characteristics (with 1:20 scale ratio applied)

The wave moment estimation data are downsampled to $10 \mathrm{~Hz}$, in order to reduce the computation burden of the prediction algorithms. The resulting 
spectra are presented in Figure 6 .
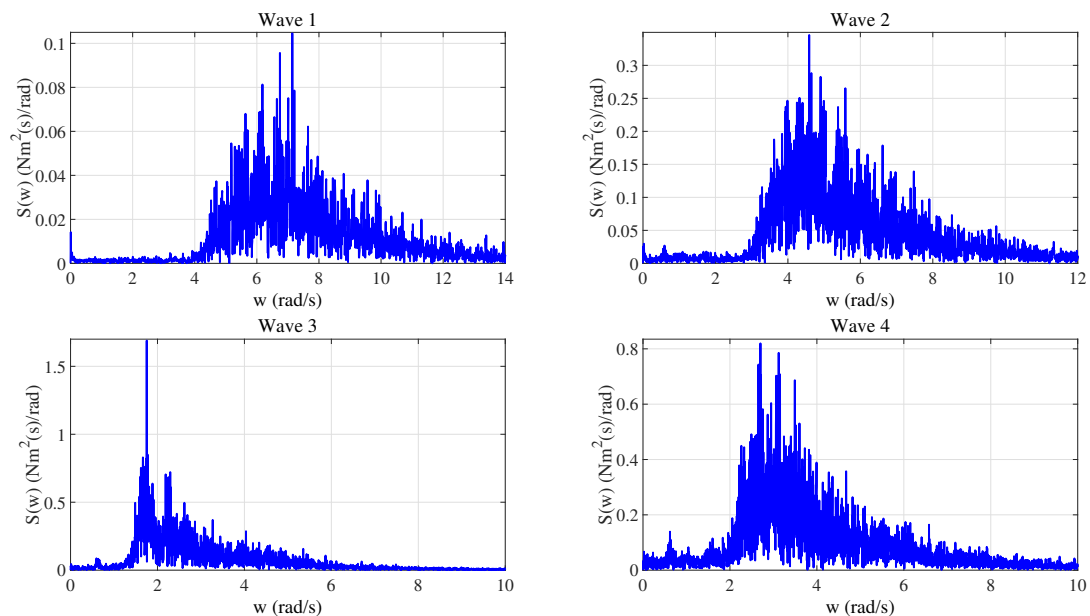

Figure 6: Wave spectra of sample data sets

Remark 5: The prediction performance corresponding to four time-invariant sea states is reported in this paper. Algorithm 1 and 2 have also been run, in real-time, with a time-varying sea state from sea state 2 to sea state 3 , see [28] for more details.

\subsection{Prediction performance}

For a given prediction horizon $N_{p}$, the following performance index is used to determine the prediction accuracy, at time $k$,

$$
M_{e}\left(N_{p}\right)=\frac{\sum_{h=1}^{N_{p}}((y(k+h)-\hat{y}(k+h \mid k)))^{2}}{M_{y}}
$$

where $M_{y}$ is the maximum of $y(k), \forall k$. It is clear that the smaller $M_{e}\left(N_{p}\right)$, the better the forecasting model. A zero value for $M_{e}\left(N_{p}\right)$ implies that the wave time series is perfectly predicted over $N_{p}$ steps into the future. Note that other indices such as mean square error, mean absolute error or mean absolute percentage error, etc, can also be used to measure the prediction accuracy [13, [19]. However they treat each horizon $h$ separately, whereas the index 35 considers all the prediction horizons together. 


\subsection{Prediction results of the EKF approach}

400 is an important factor. There is a trade-off between model complexity, which increases the computational burden to apply the EKF, and prediction performance. To guarantee a satisfactory performance, our experience shows that the order of the AR model should be greater or at least equal to $\frac{T_{p}}{T_{s}}$, where $T_{p}$ is $N_{p}=20$, is taken as baseline.

\begin{tabular}{|c|c|c|}
\hline Order & Increase in computation time & Prediction error $\mathcal{E}$ \\
\hline 20 & - & 47.1166 \\
\hline 26 & $12 \%$ & 16.8177 \\
\hline 32 & $28 \%$ & 12.6587 \\
\hline 40 & $54 \%$ & 12.4838 \\
\hline
\end{tabular}

Table 2: Trade-off between computational complexity and performance

The prediction error $\mathcal{E}$ is computed as

$$
\mathcal{E}=\sqrt{\sum_{k=1}^{N_{d}} \frac{\left(y\left(k+N_{p}\right)-\hat{y}\left(k+N_{p} \mid k\right)\right)^{2}}{M_{y}}}
$$

where $N_{d}$ is the number of data, and $M_{y}$ is the maximum of $y(k), \forall k$.

Using Table 2, it can be observed that $N_{p}=32$ is a good compromise between the performance and the computational complexity. Hence the order is set to be 32 for Algorithm 1 .

Figure 7 presents the first four parameters $a_{1}, a_{2}, a_{3}, a_{4}$ for the four waves. It can be observed that the optimal parameters $\mathbf{a}(k)$ are time-varying. This 
shows that the assumption that $\mathbf{a}(k)$ are constant is indeed restrictive. It is underlined that allowing $\mathbf{a}(k)$ to be time-varying is the way to cope with the fact that the sea state changes continuously, and that the AR model (3) is only an approximation of the real wave excitation force model.
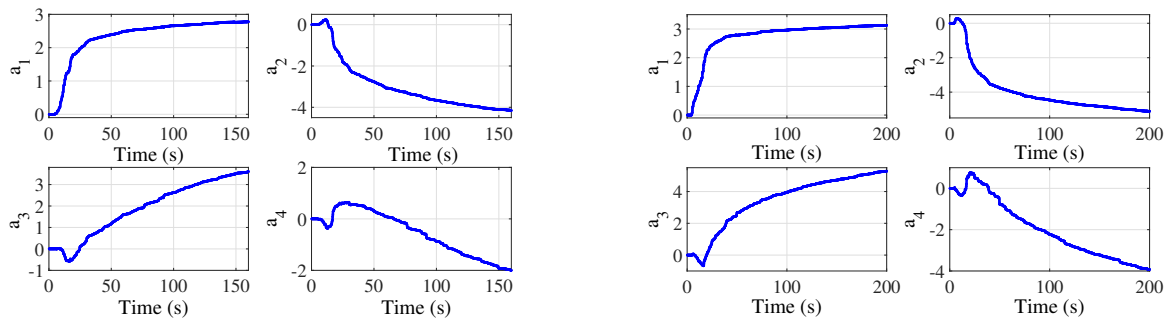

Wave 1
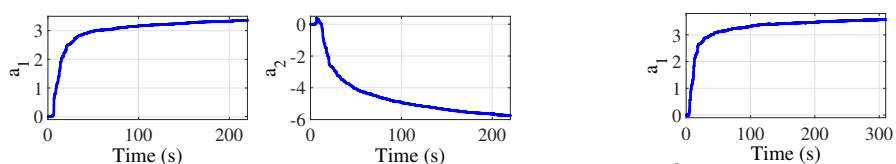

Wave 2
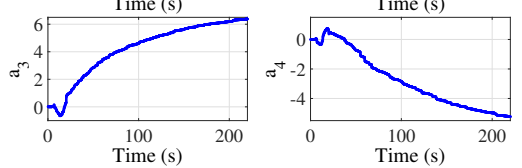

Wave 3
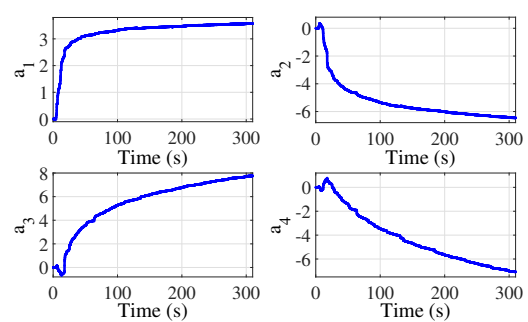

Wave 4

Figure 7: Optimal AR parameters. The time-varying nature of $\mathbf{a}(k)$ even for a time-invariant sea state is due to the fact that the AR model $\sqrt{3}$ is only an approximation of the exact model of the real system

Figure 8 shows the wave prediction performance of Algorithm 1. It can be observed that the predicted wave is in phase with the real wave. This feature 425 is indeed very important for any WEC control algorithm.

Using the performance index (35), the prediction accuracies with $N_{p}=10$ and $N_{p}=20$ for algorithm 1 and the AR based approach via the minimization of a multi-step ahead cost function [13] are compared and shown in Figure 9, It can be observed that Algorithm 1 outperforms the mentioned solution. 

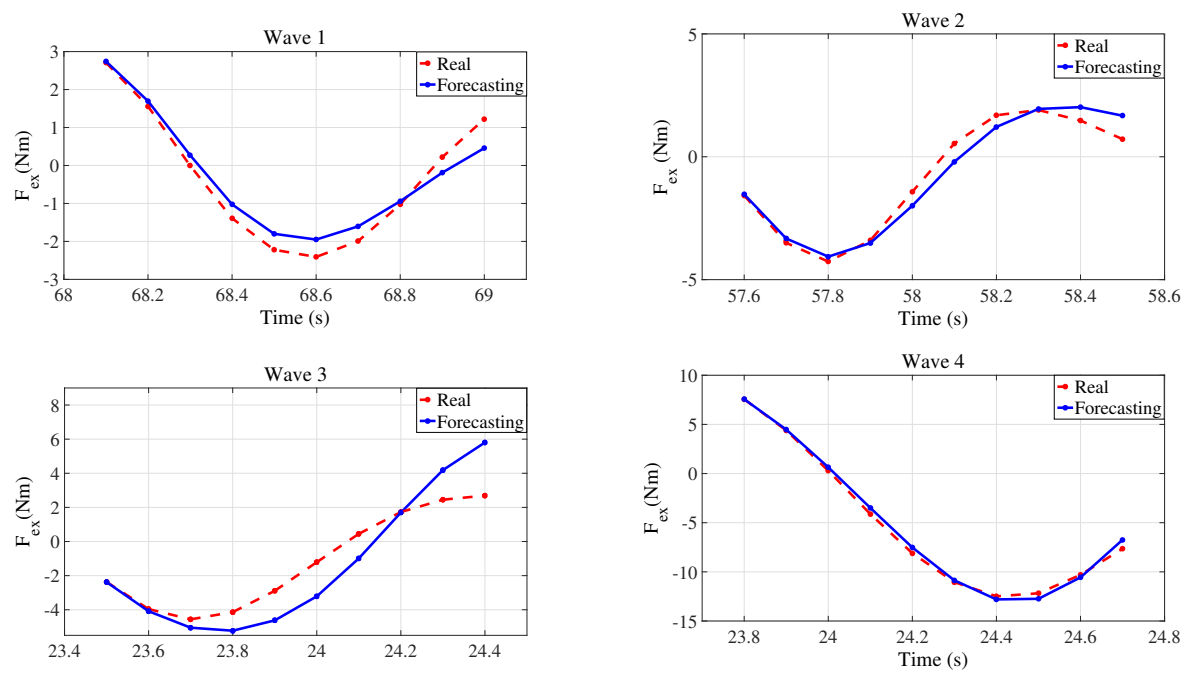

Figure 8: Wave prediction performance using Algorithm 1

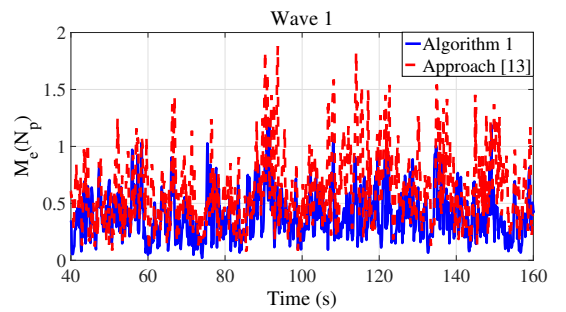

$N_{p}=10$

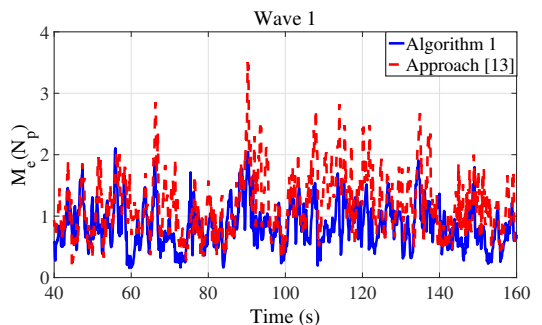

$N_{p}=20$

Figure 9: Prediction accuracy of Algorithm 1 and the approach in 13.

\subsection{Prediction results of the adaptive Kalman filter approach}

Since multiple models are used for algorithm 2, practice shows that it is enough to set the order of all the AR models to be 16 . Figure 10 presents the wave prediction accuracy of Algorithm 2. It is clear that the algorithm yields good results.

435

Finally, using the normalized error index (35), the prediction accuracy with $N_{p}=10$ for algorithm 1, and algorithm 2 are depicted in Fig. 11. Using the TIC/TOC function of Matlab 2015b, Table 3 shows the on-line computation 

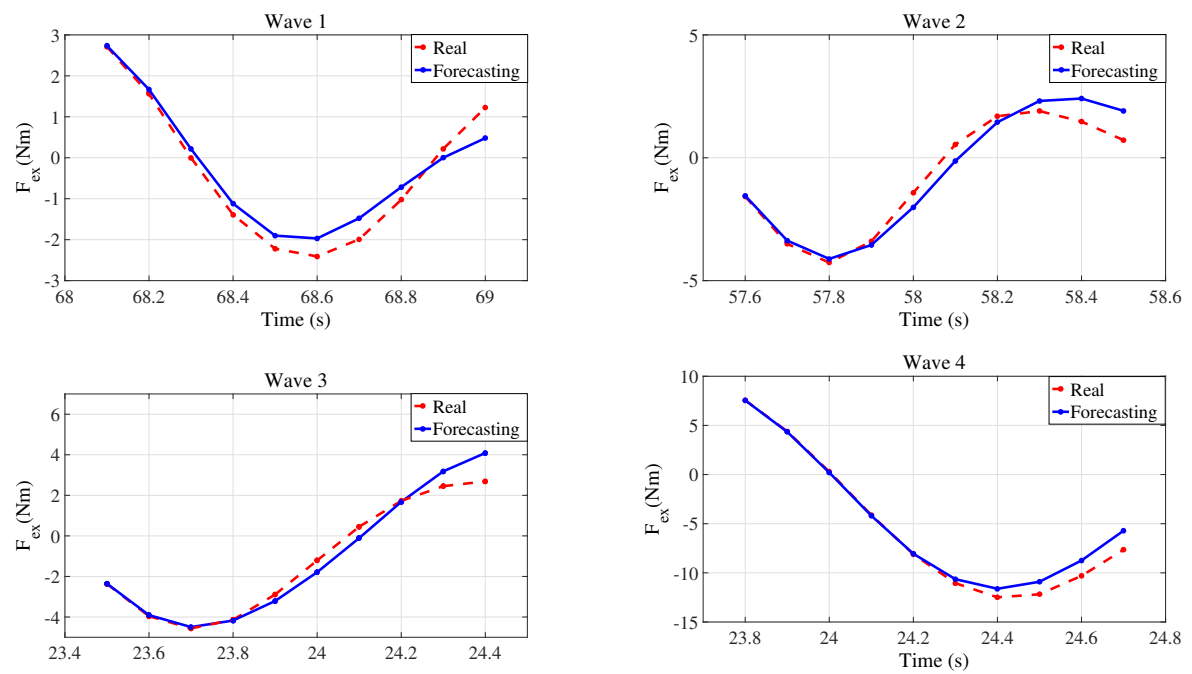

Figure 10: Wave prediction performance by using algorithm 2.

times for one discretization interval for algorithm 1 and algorithm 2. Looking at Fig. 11 and at Table 3 , it is clear that there is a trade off between performance and computational complexity. It can be observed that algorithm 2 has the highest complexity. However it yields a better performance compared to algorithm 1, which has a more modest computational complexity.

\begin{tabular}{|c|c|}
\hline & Online computation time (s) \\
\hline Algorithm 1 & 0.0228 \\
\hline Algorithm 2 & 0.0312 \\
\hline
\end{tabular}

Table 3: Duration (s) of online computations over one discretization interval for algorithm 1 and algorithm 2 .

\section{COMPUTATION OF INTERVAL FORECASTS}

Until now, wave predictions have been expressed as scalars, which one will 445 refer to as point forecasts, which do not give any idea about their likely accuracy. As a wave future value can be regarded as a random variable at the time the 

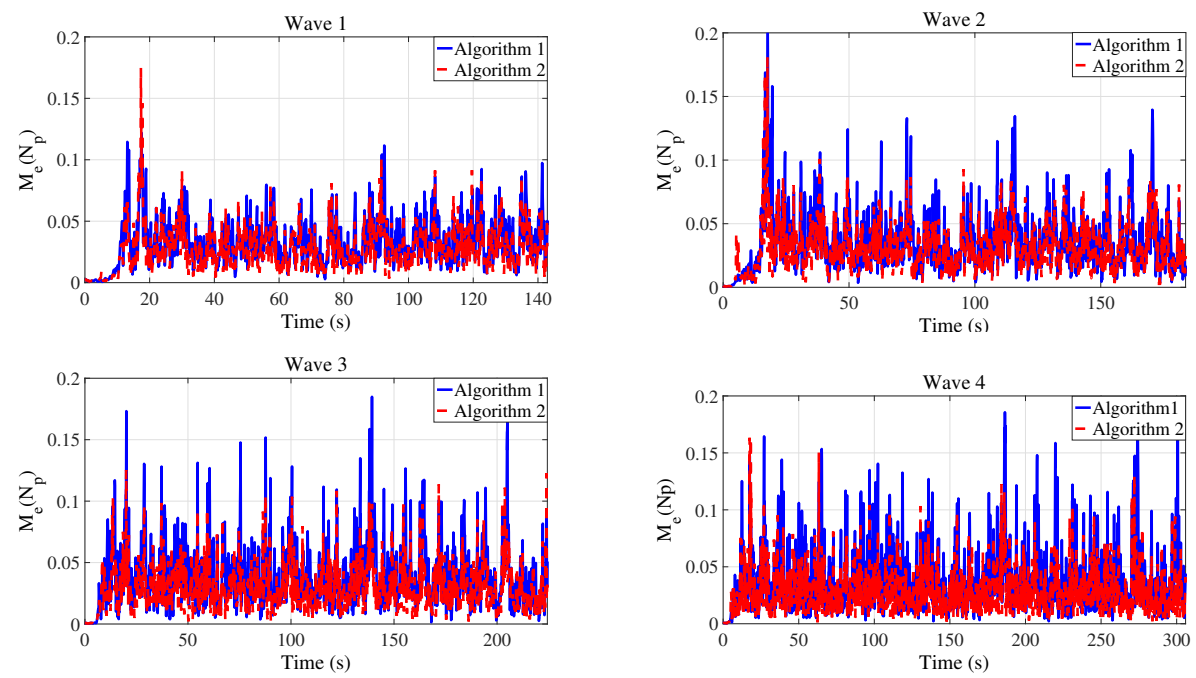

Figure 11: Prediction accuracy of algorithms 1 and 2.

forecast is made, it is more reasonable to express a wave forecast as a range of numbers, called an interval forecast. Providing point forecasts together with interval forecasts enables us to

- Assess future wave uncertainty.

- Design different control strategies for the range of possible outcomes indicated by the interval forecasts.

- Compare wave forecasts for different methods more precisely.

- Investigate different scenarios using different assumptions in more detail.

Before proceeding further, one needs to define more rigorously what does mean by an interval forecast. An interval forecast consists of an upper and a lower limit between which a future value is expected to lie with a prescribed probability. The limits are called prediction bounds or forecast limits, while the interval is sometimes called a prediction interval [29, [30, 31].

Let us assume that the $h$-step ahead prediction error

$$
\hat{e}(k+h \mid k)=y(k+h)-\hat{y}(k+h \mid k)
$$


is zero-mean Gaussian with variance $\sigma_{h}^{2}$. In the other words, the probability density function of $\hat{e}(k+h \mid k)$ is

$$
p(\hat{e}(k+h \mid k))=\frac{1}{\sqrt{2 \pi} \sigma_{h}} \exp \left(-\frac{\hat{e}(k+h \mid k)^{2}}{2 \sigma_{h}^{2}}\right)
$$

In general, as the prediction horizon $h$ is increased, the variance of the forecast error increases. In other words, the longer the lead time, the less accurate the forecast.

The probability that the error $\hat{e}(k+h \mid k)$ is contained within an interval $[-\delta, \delta]$ is calculated as follows,

$$
P\{-\delta \leq \hat{e}(k+h \mid k) \leq \delta\}=\int_{-\delta}^{\delta} p(\mu) d \mu
$$

Using (37), one obtains

$$
P\{-\delta \leq \hat{e}(k+h \mid k) \leq \delta\}=\frac{1}{\sqrt{2 \pi} \sigma_{h}} \int_{-\delta}^{\delta} \exp \left(-\frac{\mu^{2}}{2 \sigma_{h}^{2}}\right) d \mu
$$

The probability that the prediction error $\hat{e}(k+h \mid k)$ lies in the range $-\delta$ and $\delta$ with $\delta=n \sigma_{h}, n=1,2,3,4$ is given in Table 4 .

\begin{tabular}{|c|c|}
\hline$n$ & $P\left\{-n \sigma_{h} \leq \hat{e}(k+h \mid k) \leq n \sigma_{h}\right\}$ \\
\hline 1 & $68.3 \%$ \\
\hline 2 & $95.5 \%$ \\
\hline 3 & $99.7 \%$ \\
\hline 4 & $99.9 \%$ \\
\hline
\end{tabular}

Table 4: Confidence intervals

Using Table 4. it is clear that the variance $\sigma_{h}$ is all one needs to define the probability function of $\hat{e}(k+h \mid k)$ to lie in the interval $-n \sigma_{h}$ and $n \sigma_{h}$ with $n=1,2,3, \ldots$. Unfortunately, the exact value of $\sigma_{h}$ is not directly available, since the wave is not known in the figure. One way to estimate $\sigma_{h}$, at time $k$, 475 is to use the past history of the prediction errors as,

$$
\hat{\sigma}_{h}^{2}(k)=\frac{1}{k-1} \sum_{j=h+1}^{k} \hat{e}(j \mid j-h)^{2}
$$


Equation (40) uses batch-processing approach, which might be difficult to implement. An alternative way to estimate $\sigma_{h}$ is to use the following recursive equation 32

$$
\hat{\sigma}_{h}^{2}(k)=\frac{k-2}{k-1} \hat{\sigma}_{h}^{2}(k-1)+\frac{1}{k-1}(y(k)-\hat{e}(k \mid k-h))^{2}
$$

Fig. 12, Fig. 13 present, respectively, the forecasting intervals (dash-dot green), the wave excitation moment prediction (solid blue), the real wave excitation moment (dashed red) for wave 1, wave 2, wave 3 and wave 4 . These figures are obtained along with the $68.3 \%$ confidence interval. It is worth noticing that the forecasting intervals of algorithm 2 are generally smaller than the forecasting intervals of algorithm 1.
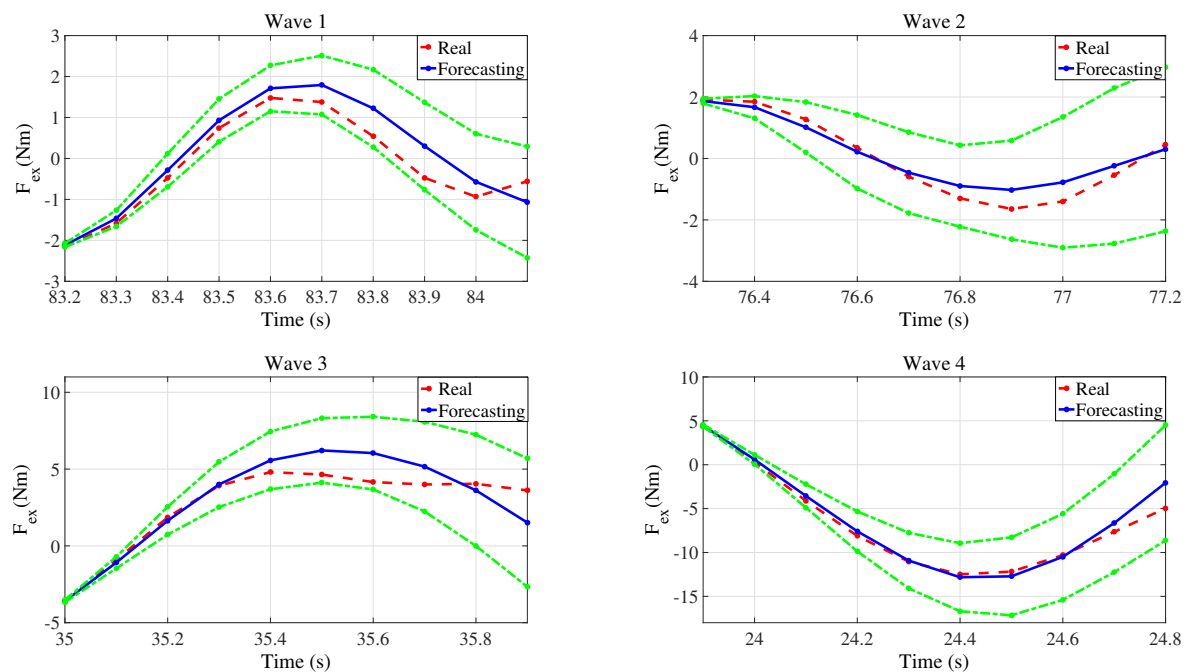

Figure 12: Forecasting intervals (dash-dot green), Wave excitation moment prediction (solid blue), Real wave excitation moment (dashed red) for algorithm 1.

\section{CONCLUSION}

The problem of short-term wave force forecasting was considered in the paper. Two new algorithms were proposed. For the set of data in this work, to improve the performance with respect to the one step ahead error minimization 

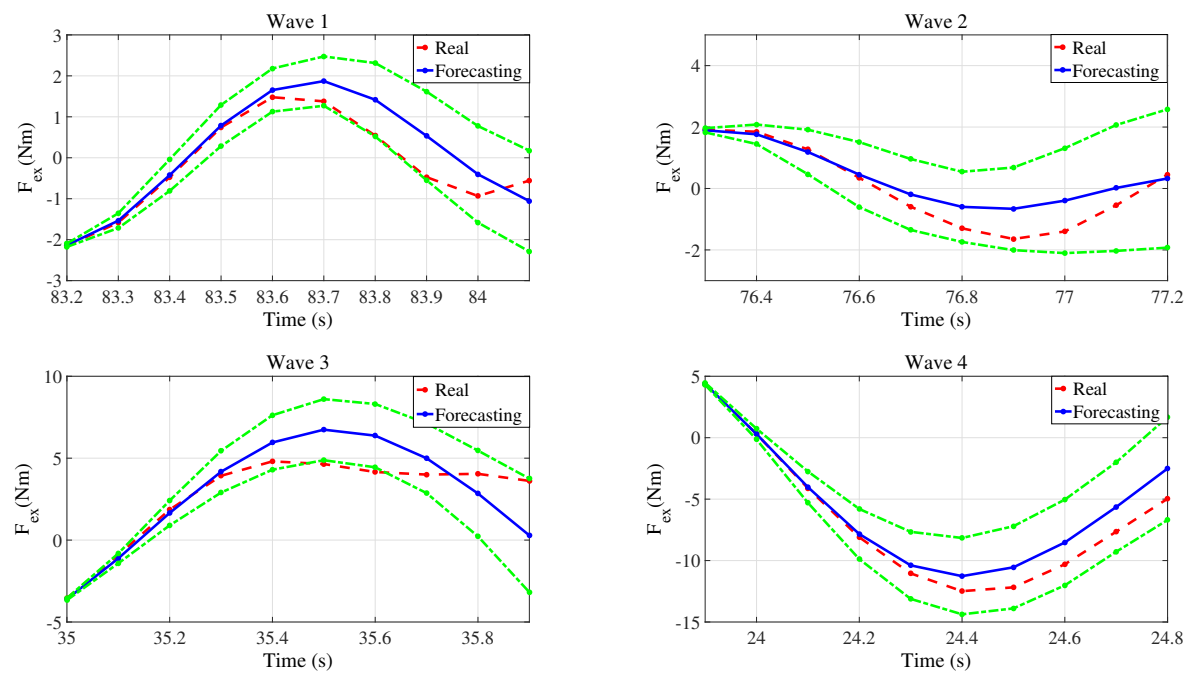

Figure 13: Forecasting intervals (dash-dot green), Wave excitation moment prediction (solid blue), Real wave excitation moment (dashed red) for algorithm 2 .

cost function, a nonlinear multi-step ahead error minimization cost function is used for the first algorithm. It is shown that the criterion adopted in [13] is a limiting case when the sea state is assumed to be constant. An extended Kalman filter (EKF) is used to obtain a solution to the nonlinear optimization problem. The main advantage of the EKF is that it is recursive and hence is easy to implement.

The second algorithm illustrates how to avoid the error accumulation problem by adopting $N_{p}$ separated models to forecast $N_{p}$ steps ahead of the wave force. The solution is also in a recursive form, since a linear Kalman filter is used for each model. It is shown that the approach in [15] is a particular case, when the sea state is constant.

Together with the wave force estimation block and the control block, the two prediction algorithms were successfully implemented on a real WEC system [28]. 


\section{References}

[1] J. Falnes, Optimum control of oscillation of wave-energy converters, International Journal of Offshore and Polar Engineering 12 (02).

[2] U. A. Korde, J. Ringwood, Hydrodynamic Control Of Wave Energy Devices, Cambridge University Press, 2016.

[3] A. Babarit, A. Clément, Optimal latching control of a wave energy device in regular and irregular waves, Applied Ocean Research 28 (2) (2006) 77-91.

[4] F. Saupe, J. Gilloteaux, P. Bozonnet, Y. Creff, P. Tona, Latching control strategies for a heaving buoy wave energy generator in a random sea, in: World Congress, Vol. 19, 2014, pp. 7710-7716.

[5] A. Babarit, M. Guglielmi, A. H. Clément, Declutching control of a wave energy converter, Ocean Engineering 36 (12) (2009) 1015-1024.

[6] G. Li, M. R. Belmont, Model predictive control of sea wave energy converters-part I: A convex approach for the case of a single device, Renewable Energy 69 (2014) 453-463.

[7] H.-N. Nguyen, P. Tona, Wave excitation force estimation for wave energy converters of the point absorber type, IEEE, 2017.

[8] J. Tedd, P. Frigaard, Short term wave forecasting, using digital filters, for improved control of wave energy converters, in: Proc. of Int. Offshore and Polar Eng. Conf, Vol. 388, 2007, p. 394.

[9] F. Serafino, C. Lugni, F. Soldovieri, A novel strategy for the surface current determination from marine $\mathrm{x}$-band radar data, IEEE Geoscience and Remote Sensing Letters 7 (2) (2010) 231-235.

${ }_{525}$ [10] F. Paparella, K. Monk, V. Winands, M. Lopes, D. Conley, J. V. Ringwood, Up-wave and autoregressive methods for short-term wave forecasting for an oscillating water column, IEEE Transactions on Sustainable Energy 6 (1) (2015) 171-178. 
[11] M. Belmont, J. Horwood, R. Thurley, J. Baker, Filters for linear sea-wave prediction, Ocean Engineering 33 (17) (2006) 2332-2351.

[12] P. Frigaard, M. Brorsen, A time-domain method for separating incident and reflected irregular waves, Coastal Engineering 24 (3) (1995) 205-215.

[13] F. Fusco, J. V. Ringwood, Short-term wave forecasting for real-time control of wave energy converters, Sustainable Energy, IEEE Transactions on 1 (2) (2010) 99-106.

[14] P.-S. Yerai, V. R. John, A critical comparison of AR and ARMA models for short-term wave forecasting, in: Proceedings of the 12th European Wave and Tidal Energy Conference, EWTEC, 2017.

[15] B. Fischer, P. Kracht, S. Perez-Becker, Online-algorithm using adaptive filters for short-term wave prediction and its implementation, in: Proceedings of the 4th International Conference on Ocean Energy (ICOE), Dublin, Ireland, 2012, pp. 17-19.

[16] J. Hals, J. Falnes, T. Moan, Constrained optimal control of a heaving buoy wave-energy converter, Journal of Offshore Mechanics and Arctic Engineering 133 (1) (2011) 011401.

[17] J. M. Jonkman, Dynamics Modeling And Loads Analysis Of An Offshore Floating Wind Turbine, ProQuest, 2007.

[18] B. George, J. G. M., R. G. C., Time Series Analysis: Forecasting And Control, Pearson Education India, 1994.

${ }_{550}$ [19] L. Ljung, System Identification: Theory For The User, Springer, 1998.

[20] D. Shook, C. Mohtadi, S. Shah, Identification for long-range predictive control, in: IEE Proceedings D (Control Theory and Applications), Vol. 138, IET, 1991, pp. 75-84. 
[21] B. Wahlberg, L. Ljung, Design variables for bias distribution in transfer function estimation, Automatic Control, IEEE Transactions on 31 (2) (1986) 134-144.

[22] R. Kashyap, Maximum likelihood identification of stochastic linear systems, IEEE Transactions on Automatic Control 15 (1) (1970) 25-34.

[23] H.-N. Nguyen, F. Guillemin, On process noise covariance estimation, in: Control and Automation (MED), 2017 25th Mediterranean Conference on, IEEE, 2017, pp. 1345-1348.

[24] P. R. Bélanger, Estimation of noise covariance matrices for a linear timevarying stochastic process, Automatica 10 (3) (1974) 267-275.

[25] B. D. Anderson, J. B. Moore, Optimal Filtering, Courier Corporation, 2005.

[26] K. J. Astrom, B. Wittenmark, Adaptive Control, Addison-Wesley Longman Publishing Co., Inc., 1994.

[27] R. E. Kalman, A new approach to linear filtering and prediction problems, Journal of Fluids Engineering 82 (1) (1960) 35-45.

[28] H.-N. Nguyen, G. Sabiron, P. Tona, M. M. Kramer, E. V. Sanchez, Experimental validation of a nonlinear MPC strategy for a wave energy converter prototype, in: ASME 2016 35th International Conference on Ocean, Offshore and Arctic Engineering, American Society of Mechanical Engineers, 2016.

[29] P. J. Brockwell, R. A. Davis, Introduction To Time Series And Forecasting, Vol. 1, Taylor \& Francis, 2002.

[30] W. William, S. Wei, Time Series Analysis: Univariate And Multivariate Methods, Addison Wesley, 1990.

[31] B. Abraham, J. Ledolter, Statistical Methods For Forecasting, Vol. 234, John Wiley \& Sons, 2009. 
[32] R. Isermann, Fault-Diagnosis Systems: An Introduction From Fault Detection To Fault Tolerance, Springer Science \& Business Media, 2006. 